\title{
PAYER PERSPECTIVES ON FUTURE ACCEPTABILITY OF COMPARATIVE EFFECTIVENESS AND RELATIVE EFFECTIVENESS RESEARCH
}

Rachael Moloney

Center for Medical Technology Policy

rachael.moloney@cmtpnet.org

\author{
Penny Mohr \\ Patient-Centered Outcomes Research Institute \\ Emma Hawe, Koonal Shah, Martina Garau, Adrian Towse \\ Office of Health Economics
}

Objectives: Our objective was to gather perspectives from payers on how comparative effectiveness research (CER) in the United States and relative effectiveness (RE) research in Europe will impact evidentiary standards for access decisions of new drugs by 2020.

Methods: We conducted semi-structured interviews with fourteen senior officials representing public and private payers, health technology assessment groups, and pricing and reimbursement bodies in the United States and Europe. An online survey assessed current use of CER/RE evidence and potential trends that might influence its use for decision making by 2020. A semi-structured interview elicited payers' definitions of CER/RE and was structured around four hypothetical cases resembling drugs expected to be more common or poised to create policy challenges by 2020. Topics included acceptance of study designs and analytic methods associated with CER/RE. A systematic content review was done to extract relevant information.

Results: According to key informants, randomization will remain an essential component for assessing comparative or relative effectiveness. They anticipate greater use of policy levers such as conditional reimbursement or prior authorization to manage diffusion of new drugs. Case studies provided important insights into situations when certain types of CER evidence may be acceptable (e.g., observational data when differences between drugs are largely convenience).

Conclusions: Industry perceptions that CER/RE will change payers' evidentiary requirements in the future are consistent with our findings. Growing investment in payers' own data and increased reliance on policy tools to control diffusion of new drugs may also influence the type of evidence industry will be required to produce by 2020.

Keywords: Comparative effectiveness research, Relative effectiveness, New drug development, Payer perspectives

Today's healthcare decision makers, including payers, patients, and doctors, face an increasingly large choice of new drug therapies and a greater need to understand the comparative safety, effectiveness and costs of available treatment options $(1 ; 2)$. Meanwhile, manufacturers are faced with a disconnect between the evidence requirements of regulatory agencies and payers, making it difficult to meet all demands. In the United States, drug efficacy trials for U.S. Food and Drug Administration (FDA) approval are usually not representative of real-world patient populations, often lacking appropriate comparators and assessing outcomes of limited relevance to payers (3-6). FDA trials can offer some insights into the relative efficacy of different drugs studied, however, regulations do not require comparative effectiveness research (CER) for new drugs (7), and the utility of results to decision makers remains unclear for studies that

This study was sponsored by a consortium of five life sciences companies: Amgen, Eli Lilly, GlaxoSmithkline, Novartis, and Sanofi. The Center for Medical Technology Policy and Office of Health Economics had full and independent authority over the entire scope of the project, including design, methods, and analysis. do compare alternative treatments (6). Evidence requirements, including appropriate comparators, are also inconsistent across European regulators, payers, and health technology assessment (HTA) bodies (8-11). While European HTA agencies still regard randomized controlled trials (RCTs) as the gold standard, many recognize some use for non-RCT data in HTA (12). As evidence requirements shift, the cost of bringing a new drug to market continues to rise (2). Simply adding CER/relative effectiveness (RE) studies on top of regulatory evidence is most likely unsustainable, and many worry the escalating costs of drug development may soon limit innovation (1). According to Schneeweiss et al. (5), the increasing demand from payers for CER evidence may slow the process of bringing new drugs to market, unless manufacturers can build in modifications to anticipate CER scenarios in the future. Given economic pressure on payers and providers to allocate health resources effectively and a growing demand for stakeholder-driven evidence, CER in the United States and RE research in Europe may play a critical role for future drug development to ensure coverage and competitive pricing of new drugs $(1 ; 3-5 ; 13)$. 
We refer to CER and RE as defined by the Institute of Medicine (14) and High Level Pharmaceutical Forum (15), respectively, but use these terms interchangeably as CER/RE throughout this study. We also consider the role of patientcentered outcomes research (PCOR), tradeoffs between different CER/RE designs, and the reliability of existing methods for adjusting for bias in observational research, which remains a major challenge to its acceptability by payers $(5 ; 16-18)$.

Our aim was to gather perspectives from a diverse group of payers on the rapidly evolving fields of CER/RE. In particular, we focused on the acceptability and use of CER/RE evidence for access decisions on new drugs in the future healthcare environments of the United States and Europe. We considered study designs and methods used or potentially useful for CER/RE, the extent to which they are used within the current paradigm of coverage decision making for new drugs, and how current trends might lead to greater (or less) acceptability by payers in 2020. We grounded our questions in hypothetical reference cases of products we expect payers to encounter frequently in the future to help identify conditions that might modify payers' evidentiary requirements.

\section{BACKGROUND}

Payers may often desire head-to-head comparisons and distrust of pharmaceutical sponsored registries, observational cohort studies, or modeling to generate relevant real-world evidence for decision making (17-19). Furthermore, the relative value and weight of different evidence used by payers in decision making varies and is not always transparent. Costs and evidence certainty can be "more likely than efficacy and safety data to influence level of formulary access" (19). Manufacturers must struggle with the inconsistent demands from different payers, as well as the widening disconnect between regulatory and payer evidentiary requirements.

Evidence requirements of European regulators are often inconsistent with those of payers and health technology assessment (HTA) bodies. The European Medicines Agency (EMA) may accept placebo-controlled or non-inferiority trials to demonstrate the quality, safety and clinical efficacy of their new medicines for marketing authorization applications $(8 ; 9)$. Payers, on the other hand, generally demand evidence that the medicine under evaluation is more effective than alternative treatment options. Regulatory agencies and payers can also express differences in opinion about endpoints, with the former accepting surrogate endpoints as valid markers and the latter preferring clinical outcomes and accepting surrogate outcomes only if clinically relevant and/or validated $(10 ; 11 ; 20)$. Evidence suggests that many HTA bodies and payers in Europe prefer head-to-head randomized trials $(20 ; 21)$. However, it also recognized that there is a place for other, non-RCT data sources (12).
In a recent survey regarding pragmatic clinical trials (PCTs), U.S. payers demonstrated limited use and varying knowledge of PCTs; however, they were interested in the realworld evidence benefits and anticipated increased acceptance of PCTs in future (22). Given payer preferences to see trial data validated in their broader, real-world patient populations, PCTs (which can be thought of as "real-world" RCTs) may be appropriate to address questions relevant to payers while still retaining high internal validity.

The evolution of Accountable Care Organizations (ACOs) may also have major implications for CER evidence demands, with a variety of new care delivery models appearing that align payments with benefits and introduce measures focused on quality of care and the reduction of overall costs. Many anticipate that payers will want to see evidence of economic value in addition to clinical value, as compared to existing alternatives $(13 ; 23)$. Although costs are excluded from the Patient Protection and Affordable Care Act of 2010, the establishment of the Center for Medicare and Medicaid Innovation placed a clear focus on improving quality and lowering costs by evaluating innovative payment and care delivery models.

Despite existing payer studies and advances in CER/RE methods, the extent to which payers anticipate how they will use CER/RE for future access decisions remains unclear. Our study attempts to address this unknown by grounding our discussion in hypothetical cases and examines specific methodologies often associated with CER/RE (e.g., indirect comparisons, cluster RCTs) to obtain perspectives about their usefulness. Importantly, however, we look to the near-future, anticipating how the environments in the United States and Europe are likely to change between now and 2020, how changes will influence payer information needs, and how CER/RE evidence may be accepted to fulfill those information needs.

\section{METHODS}

\section{Recruitment and Selection of Key Informants}

Key informants were identified from existing professional contacts and online executive/organizational leadership rosters and recruited by means of email to participate in the project. We sought senior officials representing large payers, pharmacy benefits managers (PBMs), HTA groups, and pricing and reimbursement bodies in the United States (as this represents a sizeable portion of current pharmaceutical company revenue) and Europe (in recognition of the global environment in which companies operate), who played a role in coverage and/or pricing decisions or policies in their organizations. Our initial set of European countries included the five major markets (France, Italy, Germany, Spain, and the United Kingdom) and two additional countries (the Netherlands and Sweden) where we found evidence of systematic re-evaluations based on additional data collected while a drug is used in practice (21). In the United 
Moloney et al.

Table 1. Literature Review

Description

We conducted a non-systematic literature review by searching Medline (PubMed) and the grey literature (PCORI, IOM, AHRQ, PCAST) for key papers in CER/RE methods, focusing on innovative or less common study designs, statistical methods and data sources. We also searched for primary reports of U.S. payer perspectives on CER evidence for decision making and current RE requirements from European HTA and pricing bodies. We searched key words associated with specific methods or designs (e.g. "cluster randomized trials," "indirect comparisons") individually and in combination with key words such as "payers," "coverage decisions," "drugs," "formulary," in addition to broader searches for "comparative effectiveness research methods" (or CER methods or relative effectiveness methods) and "payers" or "pricing" etc. We searched Google Scholar to identify publications in the peer reviewed and grey literature providing evidence of HTA or pricing and reimbursement requirements in 8 selected jurisdictions (England and Wales, France, Germany, Italy, The Netherlands, Scotland, Spain, and Sweden). In addition, we drew relevant content from previous work with multiple U.S. stakeholders on choosing the appropriate study designs for specific CER questions, and a review of HTA requirements across several European systems.

Table 2. Description of Hypothetical Reference Cases

\section{Hypothetical Reference Cases}

Case 1 A new drug that is a breakthrough for treating patients with a common chronic disease but has been studied only in a small population that has a specific biomarker identified by a companion diagnostic test.

Case 2 A new drug that demonstrates no or marginal difference in efficacy between alternative drugs in Phase III trials. However, the new drug uses a more convenient route of administration than existing competitors because it does not require visits to the doctor's office. This raises the possibility that patients will adhere to the new drug more than the competitors.

Case 3 A new drug in a crowded, competitive market for a common chronic disease with a demonstrated efficacy similar to its competitors. The manufacturer has identified several potential subgroups where the drug may be more effective; however, those subgroup analyses were underpowered and not planned a priori. Of the subgroups examined post hoc, one group was patients who did not improve on their initial therapies.

Case 4 A new drug for a disease where no alternative pharmaceutical treatment exists. There is a surgical procedure considered a treatment, that requires general anesthesia, and which is performed only with poor to moderate success. However, the effectiveness of the surgery has not been vigorously investigated.

Kingdom, two HTA bodies (the National Institute for Health and Care Excellence and the Scottish Medicines Consortium) operating in different jurisdictions were considered in the study as both consider cost effectiveness data in their decision making. We also recruited U.S. key informants for integrated health systems and ACOs.

\section{Online Survey and Key Informant Interviews}

To guide the development of our survey and interview plan, we reviewed key literature in CER/RE methods (Table 1). Using the Qualtrix@ online survey platform, we developed a brief survey instrument for our interviewees to complete before their interviews, assessing current use of CER/RE evidence and potential trends that might influence its use for decision making by 2020. A glossary of key terms accompanied the survey as a resource for key informants. We developed a semi-structured interview guide that could be tailored to individuals' survey responses. For both the United States and Europe, interviews were conducted by a pair of researchers (one interviewer and one note taker). Interviews were also recorded and transcribed.
Pilot

The survey was piloted for duration, ease of use, interpretability and appropriateness of questions, among three professional contacts in the United States and Canada and two in Europe who are experts in HTA, CER/RE and coverage decision making. Pilot participants then completed 60- to 90-minute cognitive interviews to share personal interpretations of the survey and semi-structured interview questions and help identify contextual elements of the hypothetical references cases (Table 2) that might create conflict or challenges for payers. Cases were revised and became the center of the semi-structured interview based on this feedback. Survey items were also modified, separated out by study designs, methods and sources of information in a series of matched "current" and "future" questions.

\section{Analysis}

Survey responses were tabulated, however individual-level changes in paired "current" and "future" questions were documented and incorporated into the semi-structured key informant interviews. We conducted a systematic content review, similar to a directed content analysis (24). Instead of in-depth 
UNITED STATES

\begin{tabular}{|c|c|c|c|}
\hline US1 & Public payer & CMS official & Involved in Medicare coverage determinations \\
\hline US2 & Public payer & State Medicaid Medical Director & $\begin{array}{l}\text { Works with pharmacy benefits manager but leads overall coverage } \\
\text { determination process }\end{array}$ \\
\hline US3 & Large private payer & VP, Hospitals, Quality \& Care Delivery & $\begin{array}{l}\text { Leads national quality and care delivery agenda, co-chairs the } \\
\text { Medicare Part D formulary }\end{array}$ \\
\hline US4 & Large private payer & Director, Health Technology Assessment & $\begin{array}{l}\text { Makes recommendations to voters on what should be considered } \\
\text { medically necessary }\end{array}$ \\
\hline US5 & Large pharmacy benefits manager & Chief Medical Officer & Leads healthcare strategy, clinical \& medical affairs \\
\hline US6 & $\begin{array}{l}\text { Large private payer }+ \text { accountable care } \\
\text { organization }\end{array}$ & $\begin{array}{l}\text { Medical Policy, Technology Assessment, and } \\
\text { Credentialing }\end{array}$ & Leads pharmacy \& therapeutics process and medical policy process \\
\hline US7 & $\begin{array}{l}\text { Large private payer }+ \text { accountable care } \\
\text { organization }\end{array}$ & $\begin{array}{l}\text { Clinical Policy Research and Development, } \\
\text { Pharmacy and Therapeutics }\end{array}$ & $\begin{array}{l}\text { Oversees clinical policies and co-chairs pharmacy \& therapeutics } \\
\text { committee }\end{array}$ \\
\hline US8 & Integrated health system & Chief Medical Officer & $\begin{array}{l}\text { Leads strategic direction of performance improvement in quality and } \\
\text { patient safety }\end{array}$ \\
\hline \multicolumn{4}{|c|}{ EUROPE: United Kingdom (including Scotland and England and Wales), Spain and Sweden } \\
\hline$\overline{\mathrm{ID}}$ & Payer perspective & \multicolumn{2}{|l|}{ Description of organization or role } \\
\hline EU1 & HTA body & \multicolumn{2}{|c|}{ Former member of Appraisal Committee, expert in Health Sciences methodology } \\
\hline EU2 & HTA body & \multicolumn{2}{|c|}{ Leadership role, Research and Development } \\
\hline EU3 & Regional pricing and reimbursement & \multicolumn{2}{|l|}{ Leadership role, Pharmacy } \\
\hline EU4 & Government Agency, Pricing \& Reimbursement & \multicolumn{2}{|l|}{ Expert in Health Economics } \\
\hline EU5 & Government Agency, Pricing \& Reimbursement & \multicolumn{2}{|l|}{ Study Coordinator } \\
\hline EU6 & HTA Body & \multicolumn{2}{|l|}{ Appraisal Committee member } \\
\hline
\end{tabular}

coding of interviews, we sought to extract explicit content in response to the questions raised during the key informant interviews. A single reviewer extracted relevant information specific to an existing framework which followed the construct of the semi-structured interview guide. The team reviewed the completed framework, discussed and resolved any disagreements.

\section{RESULTS}

Fourteen key informants from five countries participated (Table 3); of these, two completed only the interview, although their perspectives on the survey questions were gathered in the interview itself. Survey responses are available online (Supplementary Tables 1-3).

Overall, RCTs were payers' preferred source of CER/RE evidence, both generally and in response to the hypothetical reference cases. When asked to define CER in their own words, U.S. informants emphasized randomization and the use of appropriate comparators as essential aspects of CER. Efficacy RCTs were considered a potential type of CER study, depending on their usefulness, such as active-comparator trials with head-to-head comparisons of relevant clinical alternatives, or placebo-controlled trials that could be combined with other data, for example, in indirect comparisons. In general, a broad range of observational and experimental study types fit under "CER." No one found CER and PCOR to conflict, but suggested CER could be conducted without being patient-centered (e.g., comparing the effectiveness of treatment alternatives for glycemic control may be important to physicians and payers, whereas diabetes patients may be more concerned with quality of life, vascular conditions, or mortality). When asked to define RE in their own words, European key informants described similar meanings: in general, how a technology performs in practice compared to an appropriate comparator. Some pointed out that RE is the "incremental effectiveness" part of an HTA.

In the survey, payers favored direct head-to-head comparisons as "extremely relevant" to their decision making (Supplementary Table 1). The inclusion of meaningful endpoints and study populations representative of "real world" patients were "very" or "extremely" relevant to U.S. informants, and all European informants indicated that a study population being representative of real-world patients was "very" or "extremely" relevant. Table 4 summarizes high-level take-away points from the key informant interviews in response to the open-ended questions regarding study designs, methods and data sources which preceded the hypothetical reference cases. By 2020, RCTs will still be preferred for initial market access in the United States and Europe, despite improvements in observational research 
Moloney et al.

Table 4. High Level Findings from Non-Case-Specific Interview Questions

Domain: Study designs

U.S. respondents

$(n=8)$

European respondents $(n=6)$

All $(N=14)$
- Observational evidence may inform future clinical practice guidelines in U.S. by leveraging enriched databases and advanced statistics to identify observable characteristics that inform personalized medicine

- More adaptive trials expected in 2020

- U.S. payers more familiar with delayed designs

- Integrated health records and uniformity among plans will enable the conduct of these studies by payers

- More use of national registry data in Europe in the future

- More adaptive trials expected in 2020, though infrastructure issues in some countries may limit their potential

- RCTs will remain preferred study design

- Cluster RCTs being used by some (U.S. and EU) but not familiar to all

- Other potential uses of observational evidence:

- Long term safety, cost, \& effectiveness of multiple new drugs in a class compared to existing standard of care

- Identifying meaningful subgroups for which differences in treatment effect might later be confirmed by an RCT Domain: Methods/analytics

U.S. respondents $\quad$ - Large modeling groups such as Archimedes, Inc, and GNS Healthcare, may drive the sophistication and application of advanced statistics to "big data," hypothesis-free association studies, and trial simulations

- By 2020 U.S. key population based decision makers may use cost utility analyses more often

European respondents $\quad$ - Methods of adjusting for bias in RE might increase in usability with gradual acceptance of methodological standards, but current techniques still need to be improved

- Complexities in explaining these methods to high level decision makers also a concern

All $\quad$ - Bayesian statistics and advanced modeling expected to increase by 2020

- Indirect comparisons have "methodologically come of age" in both Europe and the U.S

- Still need to improve matching methodology to account for comparisons of different study populations

- May get used less if the relevance and efficiency of clinical trials improve.

Domain: Data capacity \& burden of evidence generation

U.S. respondents $\quad$ - Continued investment in the development, quality of large U.S. private payers' own databases

- New partnerships between U.S. payers and healthcare systems, between payers and independent research groups, large practice consolidations, and integration of data within single large payer systems will likely generate large amounts of clinically enriched individual-level observational datasets.

- Costs of database maintenance, proprietary, legal and oversight issues are potential barriers to sustainable data sharing infrastructures for CER

- FDA Mini-Sentinel a potential facilitator.

- Potential for misuse, such as data-mining and underreporting of negative results were concerns

- Role of manufacturers may evolve into helping develop standards and common language as other stakeholders play a greater role in CER evidence generation

European respondents $\quad$ - Increased use of national registries anticipated in Europe

- Potential shiff to greater use of electronic health records data in Europe, extent to which is unclear

- Major concerns: information technology capability issues, provision and storage of information, and some dependence Domain: Accountable care

U.S. ACO or integrated care respondents $(n=3)$

- More use of risk-adjusted per member per month payments anticipated in the future, leading to more integrated understanding of drug utilization and resource use

- Anticipated shift in performance metrics toward more sophisticated measures encompassing cost and measures of adherence and leading to more judicious decisions about use of medical care

- Clinical autonomy a potential limiting factor: physicians will continue to take individual patient preferences seriously

- Ability to evaluate drug effectiveness within ACOs will likely depend on ability to remain partnered with PBMs, who typically house the drug claims data

- ACOs may have own care pathways for which uncovered products might be absorbed if overall cost saving is achieved

- Smaller ACOs may remain focused on improving their data and developing appropriate quality measures

"For purposes of the project, we defined "big data" as a repository of a voluminous amount of unstructured or semi-structured data on diverse care settings that can be speedily accessed and the use of predictive analytics and data mining to undercover hidden patterns and unknown correlations. 
methods and a growing data-rich environment. There may be a larger role for observational study designs following initial approval, to support decisions about formulary placement and premium pricing in competitive markets. European informants also anticipated the continuing growth of adaptive trials and use of study data from registries, and considered patient reported outcomes (PROs) to be only one component of RE.

Key informants expected drugs resembling Case 1 (Table 2) to be more common by 2020 . For this case, they most often asked for an RCT or PCT in a broader population comparing outcomes of patients without a biomarker to other alternatives. Indirect comparisons, retrospective analyses of payer data, registries, or rigorous prospective observational studies were also discussed. Among U.S. key informants, the majority expressed concern about the potential for indication creep (or diffusion of the use of the new drug into broader, off-label populations) and would attempt to use available policy tools to control uptake and diffusion. European key informants focused on appropriate outcomes, that is, the acceptance of surrogate outcomes may depend on the drug's place in therapy. Consensus was that manufacturers should be responsible for generating post-launch RE evidence in Europe, although there was recognition that in some cases there are insufficient incentives to do so. Coordination with healthcare services was also mentioned. Half of the U.S. key informants would expect strong evidence to come from manufacturers, three would analyze their own data, and one would consider a partnership akin to coverage with evidence development.

Regarding Case 2 (Table 2), public and private U.S. informants emphasized that convenience was not considered medically necessary. (According to Thompson v. Western States Medical Center, 535 U.S. 357, 2002 and Section 1862(a)(1)(A) of the Social Security Act (the Act), 42 U.S.C. $1395 y(a)$ : the Secretary may only pay for items and services that are "reasonable and necessary for the diagnosis or treatment of illness or injury or to improve the functioning of a malformed body member.") Conventional, less costly alternatives would likely be preferred, without robust evidence that linked the new drug to improved clinical outcomes or (in some cases) adherence as a surrogate. This view was echoed amongst European respondents. Three U.S. and two European informants might accept registry data or other prospective observational studies instead of randomized trials in therapeutic areas where adherence was more clinically important for disease management, where disadvantages of existing alternatives (such as tolerability or patient burden) were well documented, or where the price differential between new and existing drugs was not as large. However, a key concern was that important differences would exist between the patients on different alternatives at the time of product launch, restricting the ability to compare in observational data, because the data may reside with different entities. Two U.S. key informants would look at their own claims to generate the appropriate evidence. European key informants may also ask for cost effectiveness analyses, stated preference studies, and RCTs that sought to capture adherence.

Key informants were familiar with products resembling Case 3 (Table 2) and typically stated that underpowered post hoc subgroup analyses were not convincing. Drug price, availability of generics, and unmet need were important considerations. Key informants would prefer RCT data with prospectively identified subgroups that were sufficiently powered to consider premium pricing. However, several key informants expressed a willingness to consider peer-reviewed, prospective observational cohort studies, under certain circumstances. Other key informants expressed concerns about manufacturers providing the evidence, publication bias and underpowered subgroup analyses.

Case 4 (Table 2) was considered rare, and perspectives varied, due in part to the ethical and design challenges raised by a standard of care (surgery) of questionable effectiveness. Although two European and four U.S. key informants "ideally" wanted to see a head-to-head trial, most informants recognized that the availability of evidence on the surgical procedure would influence their demands for randomization, and that an effectiveness study of the surgery might be needed first (which could be publicly funded in Europe). One U.S. informant suggested modeling or meta-analyses, and one European informant suggested identifying patient subgroups where the new treatment would be more appropriate than a surgical procedure.

Although responses to hypothetical reference cases were similar to U.S. informants, European informants expect to use more national registry data, more data collected postlaunch/HTA decision (to reduce uncertainty of pre-launch findings) and potentially from other jurisdictions. Several barriers may influence the availability of RE evidence, including: lack of funding for RE from healthcare providers, lack of incentives for industry to collect RE evidence (in many countries the access decision is made at launch), and comparators being inappropriate and different across countries. Case 1 (companion diagnostic) and Case 3 (post hoc subgroup analysis) sparked several observations about the future of personalized medicine. More targeted therapies were expected by 2020 . U.S. payers in particular anticipate a broader picture of personalized medicine enabled by the availability of clinically rich individual level payer data, analyzed in prospective real-world studies to identify observable factors that can guide decision making.

\section{DISCUSSION}

We heard from payers that RCTs will still be expected for establishing causality and providing evidence of therapeutic effect $f \circ r$ initial market access in both the United States and Europe, even with improved standards and data quality. Payer preferences for appropriate comparators and information on real-world patients support greater use of pragmatic trials before market release in future drug development. However, whether or not PCTs can 
become an efficient design by leveraging existing data is somewhat uncertain. Many U.S. payers anticipate a significant growth in the availability of electronic data for CER evidence generation, with implications for multi-stakeholder sharing of the burden of CER/RE evidence development. However, European payers expressed concerns that poor health system infrastructure may be a barrier to electronic health records reaching their full potential. On the other hand, investments by payers, health care systems and countries in electronic health data and registries may facilitate delayed design studies and retrospective claims analyses to examine use in covered populations and refine formularies (for example, delayed design studies might resemble randomized or staggered implementation of policies within a covered population to compare drug usage and health outcomes). We might also anticipate a growing role of observational study designs following initial approval to support decisions about formulary placement and premium pricing in competitive markets. As evidenced by reactions to the hypothetical reference cases, although payers/HTA bodies may remain circumspect about observational studies or data mining conducted by the pharmaceutical industry, such studies may be acceptable for certain products, under certain contexts. Like Wang et al. (17), we found that (large) U.S. payers express comfort with their own claims data, which are increasingly being enriched with clinical and laboratory data to provide good quality data for observational research. The use of novel designs may also reduce concerns about bias in peer-reviewed observational studies on newly marketed drugs (5). For example, inception cohorts or new user designs may help avoid biased comparisons of new users versus prevalent users of a drug, who may differ in several important ways (25).

Manufacturers may benefit from investing in infrastructure for post-market follow-up that anticipates greater competition over time. A panel of nine public and private U.S. payers involved in formulary decision making preferred long term cost and outcome data and acknowledged the appropriateness of well-designed observational studies to complement RCT findings in broader populations (3). These same payers also recommended that manufacturers validate trial findings in real world studies to show payers that outcomes in their plans' membership reflect RCT findings (3). Epstein suggests more collaboration in analyzing findings, and shared access to raw data including those from clinical studies (4). Schneeweiss et al. (5) suggest a multifaceted approach given the lack of early observational data and limitations of electronic health records (EHRs), registries, and claims data. They propose sequential cohort designs, the extension of phase III and IV trials (i.e., patients are followed for longer, perhaps using an observational design), indirect comparisons of efficacy, and the use of modeling and trial simulation. Like several of our key informants, they see an accelerated trend toward third-party generation of CER/RE evidence that leverages longitudinal claims and EHR data from payers and healthcare systems.
In a future healthcare environment most conducive to CER/RE, where PCT enrollment is facilitated by EHR enriched environments, access to patient registries and manufacturer partnerships with advocacy groups or healthcare systems, the drug development paradigm might rely heavily on PCTs with extended observational follow-up, including sequential cohort studies and registries. However, a less integrated scenario where electronic data offer some but not comprehensive benefits, may be more likely. Still, new platforms for research offer partnership opportunities for manufacturers seeking more efficient ways to conduct RCTs. Health systems investments and population-based registries offer a basis for efficiently identifying trial populations and collecting clinical trial data using routine data sources. The implications for evidence collection during drug development may depend heavily on whether a product is being marketed broadly in a crowded market but seeking special status, versus being marketed to a targeted population, but with the potential to benefit other groups who are not on label. A major challenge for companies in the competitive "me too" scenario (Case 4) is the market competition that cannot be predicted at start of a product portfolio: companies often end up in a crowded market, but not by design. Improving the efficiency of evidence generation while maintaining validity is key, but a product portfolio may need to allow a certain amount of flexibility to adapt to emerging competitors (e.g., add study arms or other outcomes), whereas historically many manufacturers might have scrambled toward the end of a product's evidence development to deal with this competition. Companies may also want to consider the future of exploratory research and how the decision point regarding a product's continuance or discontinuance within a company's development portfolio might shift.

The capabilities of European countries and the United States currently differ; how each healthcare environment evolves between now and 2020 has implications for healthcare systems capabilities and data availability, which may in turn influence the ability to generate CER/RE evidence in different regions. For example, Europeans healthcare systems have longitudinal data on patients over the course of care, whereas the U.S. healthcare system is disconnected, but a wealth of electronic data from EHRs and other databases exist. This may explain the greater emphasis on registries among our European informants in contrast to the demand for randomized studies from our U.S. informants.

Surprisingly, there was little mention by key informants about an increased role of coverage with evidence development, except in the pilot discussions. However, we heard repeatedly from the U.S. payers we interviewed that they will increasingly attempt to restrict access through prior authorization, step-therapy, or other policy levers. As data accumulate or classes become crowded, they will re-evaluate outcomes in their own data, a re-iterative process aimed at refining formularies. Some European informants thought the evidence received at launch would depend on whether the EMA changes its approval process. The studies payers accept may depend on additional 
evidence the EMA is seeking post-launch as part of an adaptive licensing process. Should a product get early marketing authorization, there may be a political pressure to provide the product within that country's health system; this may require greater use of post-launch studies, perhaps linked to some form of conditional reimbursement.

\section{Limitations}

Our study is limited by a small sample size. Only six of fourteen European key informants agreed to participate. Perhaps the inclusion of more advisors to decision makers rather than officials representing the selected decision-making bodies could have increased the response rate. In addition, some European countries included in the final sample are over-represented (two experts from Sweden and three from the United Kingdom, although in the latter informants were from different jurisdictions). Those countries rely primarily on cost effectiveness assessments to make coverage decisions which is not the case in other countries (i.e., Germany and Italy, which not included in the study due to non-response). Further studies including broader spectrum of European countries might provide additional insights on the future use of RE.

\section{CONCLUSION}

Our study offers insights into the evidence demands manufacturers might anticipate for market access in future healthcare environments. In the most likely future environment, manufacturers will still need to go distinctly for regulatory approval. However, more pragmatic Phase III trial designs may be increasingly feasible and affordable. Next steps could involve engaging payers, manufacturers and regulatory agencies in discussions regarding key methodological tradeoffs. From a manufacturer perspective, greater emphasis on post launch investments may first require more clarity on what returns on investment they can expect. Payers may contribute by communicating back to manufacturers clear ways in which evidence will have implications for decisions.

\section{SUPPLEMENTARY MATERIAL}

Supplementary Tables 1-3

http://dx.doi.org/10.1017/S0266462315000203

\section{CONFLITS OF INTEREST}

The authors would like to acknowledge funding support for this project from the following sponsors: Amgen, Eli Lilly \& Company, GlaxoSmithKline, Novartis, and Sanofi-Aventis. The authors have no other relevant affiliations or financial involvement with any organization or entity with a financial interest in or financial conflict with the subject matter or materials discussed in the manuscript apart from those disclosed.

\section{REFERENCES}

1. President's Council of Advisors on Science and Technology. Report to the President on propelling innovation in drug discovery, development, and evaluation. September 2012. http://www.whitehouse.gov/sites/ default/files/microsites/ostp/pcast-fda-final.pdf (accessed November 8, 2012).

2. Mestre-Ferrandiz J, Sussex J, Towse A. The R\&D cost of a new medicine. (December 2012). London: Office of Health Economics.

3. Oderda G, Sifford-Wilson SM. Bringing Liraglutide to market: A CER case study. J Manag Care Pharm. 2012;18:S12-S18.

4. Epstein RS. The hypothetical migraine drug comparative effectiveness study: A payer's recommendations for obtaining more useful results. Health Aff (Millwood). 2012;31:2225-2230.

5. Schneeweiss S, Gagne JJ, Glynn RJ, Ruhl M, Rassen JA. Assessing the comparative effectiveness of newly marketed medications: Methodological challenges and implications for drug development. Clin Pharmacol Ther. 2011;90:777-790.

6. Chokshi DA, Avorn J, Kesselheim AS. Designing comparative effectiveness research on prescription drugs: Lessons from the clinical trial literature. Health Aff (Millwood). 2010;29:18421848 .

7. Edwards CC. Hearing regulations and regulations describing scientific content of adequate and well-controlled clinical investigations. Fed Regist. 1970;35:7250-7253.

8. Schoonveld E. Think like a payer. Pharmaceutical market Europe. http://www.pmlive.com/pharma_news/think_like_a_payer_275432 (accessed March 14, 2013).

9. Garattini S, Bertele' V. Debate: Comparative clinical effectiveness. Eurohealth. 2009;15:4-11.

10. Shah KK, Mestre-Ferrandiz J, Towse A, Smyth EN. A review of health technology appraisals: Case studies in oncology. Int $J$ Technol Assess Health Care. 2013;29:101-109.

11. Kleijnen S, George E, Goulden S, d'Andon A, et al. Relative effectiveness assessment of pharmaceuticals: Similarities and differences in 29 jurisdictions. Value Health. 2012;15:954-960.

12. Wilsdon T, Serota A. A comparative analysis of the role of impact of health technology assessment. Report for EFPIA, PhRMA, Medicines Austrlia and EuropaBio. London: Charles River Associates; 2011.

13. Nelleson D, Birnbaum HG, Greenberg PE. Perspectives on comparative effectiveness research: Views from diverse constituencies. Pharmacoeconomics. 2010;28:789-798.

14. Sox HC, Greenfield S. Comparative effectiveness research: A report from the Institute of Medicine. Ann Intern Med. 2009;151:203205.

15. European Commission Enterprise and Industry Health Care Industries Pharmaceutical Forum. http://ex.europa.eupharmaforum/ docs/rea_principles_en.pdf (accessed March 14, 2013).

16. Watkins JB. Payer perspectives on health technology assessment. $J$ Manag Care Pharm. 2012;18:265-267.

17. Wang A, Halbert RJ, Baerwaldt T, Nordyke RJ. US payer perspectives on evidence for formulary decision making. J Oncol Pract. 2012;8:22s$27 \mathrm{~s}$.

18. Leung MY, Halpern MT, West ND. Pharmaceutical technology assessment: Perspectives from payers. J Manag Care Pharm. 2012;18: 256-264.

19. Dean BB, Ko KJ, Graff JS, et al. Transparency in evidence evaluation and formulary decision-making: From conceptual development to real-world implementation. P\&T. 2013;38:465-483.

20. Levy ARR. International comparison of comparative effectiveness research in five jurisdictions: Insights for the US. Pharmacoeconomics. 2010;28:813-830. 
Moloney et al.

21. Sorensen C. Use of comparative effectiveness research in drug coverage and pricing decisions: A six-country comparison. Issue Brief (Commonwealth Fund). 2010;91:1-14.

22. Ratner J. Health care payers' views of PCT features and trade-offs: A preliminary look. International Society for Pharmacoeconomics and Outcomes Research 2010 Annual Meeting, Atlanta. http://www.ispor. org/meetings/atlanta0510/presentations/IP5-RatnerJonathan.pdf (accessed November 8, 2012).

23. Brixner DI, Watkins JB. Can CER be an effective tool for change in the development and assessment of new drugs and technologies? J Manag Care Pharm. 2012;18:S6-S11.

24. Hsieh H-F, Shannon SE. Three approaches to qualitative content analysis. Qual Health Res. 2005;15:1277-1288.

25. Schneeweiss S. A basic study design for expedited safety signal evaluation based on electronic healthcare data. Pharmacoepidemiol Drug Saf. 2010;19:858-868. 